\title{
A Simplified Model of the Internal Combustion Engine
}

Christofer Neff

University of South Florida

\author{
Advisors: \\ Arcadii Grinshpan, Mathematics and Statistics \\ Scott Campbell, Chemical \& Biomedical Engineering \\ Problem Suggested By: Scott Campbell
}

Follow this and additional works at: https://digitalcommons.usf.edu/ujmm

Part of the Heat Transfer, Combustion Commons, and the Mathematics Commons

UJMM is an open access journal, free to authors and readers, and relies on your support:

Donate Now

\section{Recommended Citation}

Neff, Christofer (2013) "A Simplified Model of the Internal Combustion Engine," Undergraduate Journal of Mathematical Modeling: One + Two: Vol. 5: Iss. 1, Article 5.

DOI: http://dx.doi.org/10.5038/2326-3652.5.1.5

Available at: https://digitalcommons.usf.edu/ujmm/vol5/iss $1 / 5$ 


\title{
A Simplified Model of the Internal Combustion Engine
}

\begin{abstract}
This project further investigates a model of a simplified internal combustion engine considered by Kranc in 1977. Using Euler's method for ordinary differential equations, we modeled the interaction between the engine's flywheel and thermodynamic power cycle. Approximating with sufficiently small time intervals ( 0.001 seconds over a period of 12 seconds) reproduced Kranc's results with the engine having an average angular velocity of $72 / \mathrm{sec}$.
\end{abstract}

Keywords

internal combustion engine, thermodynamics, angular velocity 


\section{PROBLEM STATEMENT}

Recreate a manageable model of a two-stroke internal combustion engine in order to demonstrate the possible fluctuations that present themselves due to a varying distribution of power and load simplified by the use of a Scotch yoke linkage for the connecting rod (Figure 1). Upon modeling the mechanical system, the results provided by Kranc in the IJMEE journal were replicated for the internal combustion engine with an approximate average angular velocity of 72 $\mathrm{s}^{-1}$. This accounts for fluctuations in the angular velocity over time, assuming the engine is operating on an Otto cycle using air as the working fluid. If the mass of the flywheel is sufficiently small, prominent fluctuations in the angular velocity corresponding to the power cycle will be apparent. The interaction between the flywheel and the thermodynamic power cycle may be demonstrated by numerical solutions of the governing equations.

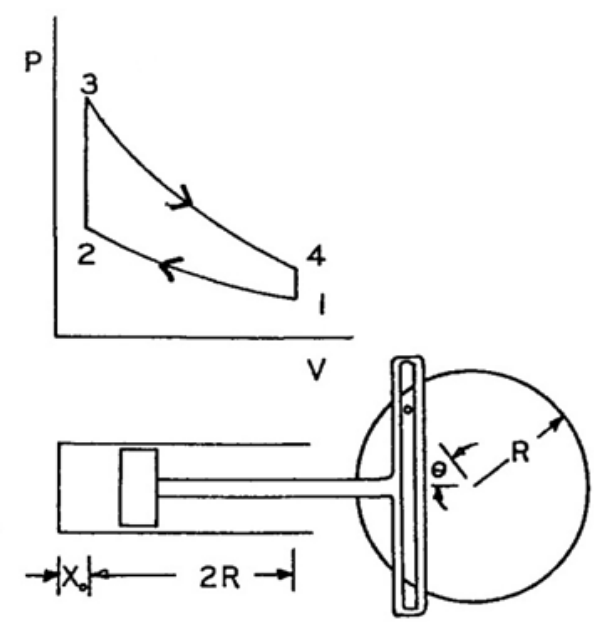

Figure 1: Simplified engine and cycle [Extracted from (Kranc 1977)] 


\section{MOTIVATION}

Machines have purpose, and that purpose is to serve as a tool to achieve a specific goal or end. Modeling systems of machines is essential to most fields of engineering and research ranging from medicine, media, mechatronics, databases, and even tools we use constantly such as the internet. Mathematical analysis allows engineers and scientist to intelligently predict the performance of a machine based on its approximate specifications. The objective of this project is to recreate the given model of a simplified internal combustion engine to a suitably small approximation using Euler’s method for ordinary differential equations.

\section{MATHEMATICAL DESCRIPTION AND SOLUTION APPROACH}

Before discussing the mathematics of the problem it is important to understand the concepts behind the movement of a two-stroke internal combustion engine. The two factors which most affect the movement of the two-stroke internal combustion engine are: 1) the working fluid and the dimensions relative to the piston or flywheel and 2) the relationship between the engine’s temperature, pressure, and volume.

Friction and the weight of parts, such as the connecting rod of the piston, are also relevant to the model, however these factors will be negated due to the use of air as the working fluid and the assumption that the connecting rod is massless or light enough to be ignored. In this case, conceptualizing the system is vital and best represented by Figure 1 as a reference.

Beginning with the movement of the engine, two primary and related movements are modeled by the fundamental equations of this project. The first being the movement of the scotch yoke linkage which converts translational or straight motion into rotational motion. Looking at 
Figure 1, this piece is shaped as a hollow rectangle attached to a pin and allows a straighter more direct flow of power from the flywheel which is the circular portion. Without the scotch yoke linkage power would not be delivered as cleanly causing greater variations and less efficient data. Variables relative to this movement are radius of the flywheel denoted by $R=0.305 \mathrm{~m}$ and the crank angle $\theta$.

For this model, the crank angle is taken in radians, varying from $0<\theta<2 \pi$. Since the stroke position directly corresponds to the position of the flywheel, the crank angle determines whether the engine is on a compression stroke or a combustion stroke. The beauty of the twostroke engine is that in one revolution a full power cycle is taken whereas a four-stroke engine takes 2 revolutions to complete a full power cycle. For this engine, a break between the compression stroke and the combustion stroke is what will cause the final graph to fluctuate in a wave-like pattern. The final graph introduced in Kranc’s journal entry is as follows:

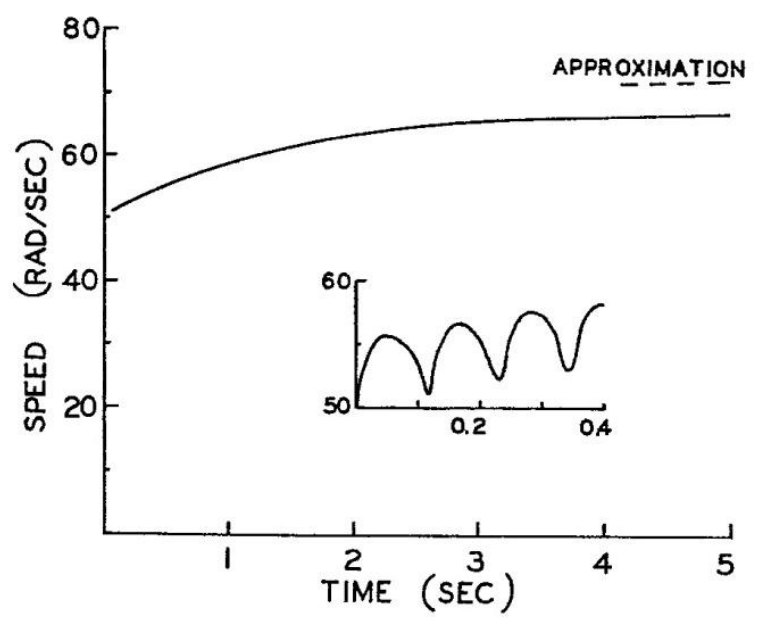

Figure 2: Simplified Engines cycle power output of $72 \mathrm{rad} / \mathrm{sec}$ [Extracted from (Kranc 1977)] 
The second kind of movement pertaining to the two-stroke internal combustion engine is the movement of the piston which deals with the working fluid, pressure/volume/temperature, and the dimensions within the piston. Referring once again to Figure $1, x_{0}=0.0254 \mathrm{~m}$ is the clearance distance better referred to in this case as the compression possible during the compression stroke of the Otto cycle. At this maximal point, the spark plug will ignite beginning the second stroke better known as the combustion stroke. A central point of focus is the fact that air is used as the working fluid, as by doing so friction is minimized to a great degree and a clear relationship can be drawn between pressure, volume, and temperature given that air is an ideal gas.

In regard to air, the benefit of having this fluid as the working fluid is that its specific heat is known. It is beneficial since this system happens to be an isentropic system of compression and expansion meaning the entropy of the system remains constant, i.e., it is a polytropic process such that

$$
P V^{n}=C
$$

where $P$ is the pressure, $V$ is the volume, $n$ is the polytropic index of the process, and $C$ is some constant. With this in mind, the working fluid allows the thermodynamic manipulations of the ideal gas law and specific heat to return a polytropic index of $n=1.3$. By taking this approach, the pressure, volume, and temperature are constant throughout the system.

Presently, a link can be drawn between the movements of the flywheel and the piston. In essence, they are respectively the dynamics of the internal combustion engine and the interaction of the flywheel and the thermodynamic power cycle. By noting that the power cycle is formulated from the combustion and compression stroke, the fundamental equation governing 
this system comes to light. In particular, the fundamental equation is derived by summing the moments of inertia on the flywheel assuming the connecting rod is massless (Kranc 1977). At this point we model the torque inhibiting movement caused by the weight present as well as the torque from a pressure force

$$
I \ddot{\theta}=T_{\text {piston }}-T_{\text {load }}
$$

where $I=3.171 \mathrm{~kg} \mathrm{~m}^{2}$ is the moment of inertia, $\ddot{\theta}$ is angular acceleration or second derivative of $\theta, T_{\text {piston }}$ is the torque of the piston, and $T_{\text {piston }}$ is the torque of the load accomplished by a Scotch yoke linkage which converts translational motion into rotational motion. In the ideal case, the motion of the piston would be purely sinusoidal with a uniform angular velocity, but this is not the case in most real world applications. To simulate real world application, a massless connecting rod and scotch yoke linkage are used as fluctuations are prominent when the flywheels mass is sufficiently small.

A constant pressure force acts on the pistons area of $A=0.001883 \mathrm{~m}^{2}$, and acts as a moment arm of $R \sin \theta$ where $R=0.305 \mathrm{~m}$ is the radius of the flywheel and $\theta$ is the crank angle. The constant force will affect the torque of the piston or rather take its place, however the torque of the load is affected by the angular velocity of the piston along with a load constant $C=0.0113 \mathrm{kgm}^{2}$. Additionally, the torque of the load is modified as it is proportional to the square of the angular velocity changing (1) to,

$$
I \ddot{\theta}=P A(R \sin \theta)-C \omega^{2}
$$

Modifying (2) further in order to calculate the pressure $P$, it is assumed that the engine is operating on an Otto cycle using air as the working fluid. Ideal gas law variables of pressure, volume and temperature are to be kept constant during the power cycle. Exceptions only apply to 
the dependent and independent variables along with any changes in throttle; modification in speed ignored. Cylinder pressure during compression is then given with a polytropic index of $n=1.3$ as an exponent to account for temperature with pressure at this cycle being $P_{1}=$ 0.1MPa. At expansion the polytropic index is also used, yet the pressure will be for that of expansion is $P_{3}=10.3 \mathrm{MPa}$. The volume of the cylinder at any stroke is represented by the relationship of,

$$
V=\left(R-R \cos \theta+x_{o}\right) A
$$

with $x_{o}=0.0254 m$ being the clearance distance, and the moment arm of volume is evaluated at two points for each stroke. With this in mind a ratio is given for volume at each stroke being the beginning crank angle over the current crank angle at some specific point denoted by time. Then, the fundamental equation comes to light by uniting (2) and (3) and transferring the moment of inertia on the flywheel to the right:

$$
\ddot{\theta}= \begin{cases}P_{3}\left(\frac{R-R \cos \theta_{1}+x_{o}}{R-R \cos \theta+x_{o}}\right)^{n} \frac{A R \sin \theta}{I}-\frac{C \omega^{2}}{I}, & 0<\theta \leq \pi \\ P_{1}\left(\frac{R-R \cos \theta_{3}+x_{o}}{R-R \cos \theta+x_{o}}\right)^{n} \frac{A R \sin \theta}{I}-\frac{C \omega^{2}}{I}, & \pi<\theta \leq 2 \pi\end{cases}
$$

In order to complete the model, we take the values for $\theta_{1}<\theta<\theta_{2}$ and input $\pi<\theta<$ $2 \pi$ and for $\theta_{3}<\theta<\theta_{4}$ respectively becomes $0<\theta<\pi$. The reason $\theta_{1}$ begins at a value greater than zero is that the Otto cycle in this case begins at the intake stroke or combustion stroke meaning the flywheel is at $\pi$. Now that we have all of our variables to the right side of the equation and angular acceleration on the left side, what is left to do is simplify for angular velocity from angular acceleration. 
At this point (4) it is a non-linear second order ordinary differential equation, hence, Euler's method can be applied. If we can apply this method we need to define which variables are dependent so they can be used as some set of initial conditions to be implemented for a straight forward solution. Considering the designations of differential equations, we know this is a second order differential equation by taking note of the highest order derivative, in this case $\ddot{\theta}$, is non-linear by the trigonometric functions present. A simple numerical scheme such as Euler's modified method with some initial conditions can be used to obtain a particular solution. Given $\omega, \theta, t$ are the dependent variables or unknowns and the rest of the values in (4) are known, we start $\omega$ at $50 s^{-1}$ and $\frac{\theta}{t}$ at 0 (refer to Table 1 for values).

For a visual aid, we can assume $\omega^{\prime}$ or the derivative of angular velocity is equal to $\ddot{\theta}$ or angular acceleration and each equation is split into its respective two-stroke power cycle with $\omega^{\prime} a$ and $\omega^{\prime} b$. From there, two first order equations each with some initial conditions were chosen to properly simplify this second order differential equation. The initial conditions used were angular velocity $\omega=50 \mathrm{~s}^{-1}$, crank angle $\theta=0$, and time $t=0.001 \mathrm{~s}$. Thus, we break down our equation to solve for a function of $\omega$ and $\theta$.

$$
\omega^{\prime}=\ddot{\theta}= \begin{cases}\omega^{\prime} b=P_{3}\left(\frac{R-R \cos \theta_{1}+x_{o}}{R-R \cos \theta+x_{o}}\right)^{n} \frac{A R \sin \theta}{I}-\frac{C \omega^{2}}{I}, & 0<\theta \leq \pi \\ \omega^{\prime} a=P_{1}\left(\frac{R-R \cos \theta_{3}+x_{o}}{R-R \cos \theta+x_{o}}\right)^{n} \frac{A R \sin \theta}{I}-\frac{C \omega^{2}}{I}, & \pi<\theta \leq 2 \pi\end{cases}
$$

Since the second derivative of angular position $\theta$ is equal to the first derivative of angular velocity $\omega$, angular acceleration is given by $\omega^{\prime}$.

$$
\frac{d \theta}{d t}=\omega \Rightarrow \theta^{\prime}=\omega
$$

Setting up our equation for our dependent variables yields, 


$$
\omega_{\text {new }}=\omega_{\text {previous }}+\Delta t \omega_{\text {previous }}^{\prime}
$$

and,

$$
\theta_{\text {new }}=\theta_{\text {previous }}+\Delta t \theta_{\text {previous }}^{\prime}
$$

Equations (5), (6), (7), and (8) can be tabulated to solve for the unknowns and see how the rate of change relates these variables (refer to Table 3 given in Appendix for numerical results).

Essentially, the smaller the time intervals used to test the differential equation, the better the result. Therefore $\Delta t=0.001 \mathrm{~s}$ time intervals were used.

\begin{tabular}{|rrrrrrr|}
\hline \multicolumn{1}{|c}{ Trial } & \multicolumn{1}{c}{$\mathbf{c}$} & \multicolumn{1}{c}{$\boldsymbol{\theta}$} & \multicolumn{1}{c}{$\boldsymbol{\boldsymbol { \theta } ^ { \prime }}$} & \multicolumn{1}{c|}{$\boldsymbol{\omega}^{\text {'a }}$} & \multicolumn{1}{c|}{$\boldsymbol{\omega}^{\mathbf{~ ' b}}$} \\
\hline $\mathbf{1}$ & 0 & 0 & 50 & Eq.(6) & Eq. (5)a & Eq. (5)b \\
\hline $\mathbf{2}$ & 0.001 & Eq.(8) & Eq. (7) & 49.991 & 49.440 & 82.542 \\
\hline $\mathbf{3}$ & 0.002 & 0.100 & 50.074 & 50.074 & 101.214 & 163.705 \\
\hline $\mathbf{4}$ & 0.003 & 0.150 & 50.237 & 50.237 & 141.948 & 227.581 \\
\hline
\end{tabular}

Table 1: First four iterations of Euler's method used to approximate (6)

An Excel spreadsheet was used to carry out calculation up to 12 seconds. Microsoft Excel graph was then generated to replicate Kranc's results (see Figures 3 and 4 below).

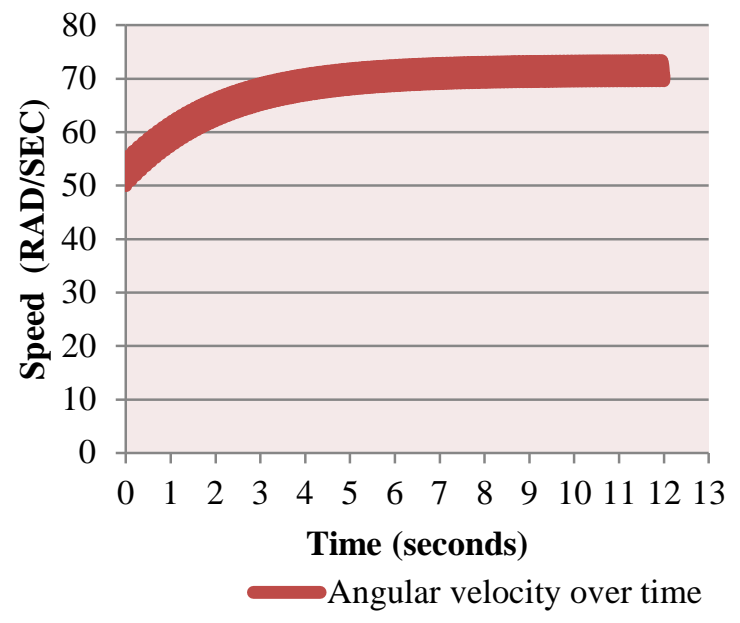

Figure 3: Primary chart used to graph angular velocity against time of some 12,000 cells.

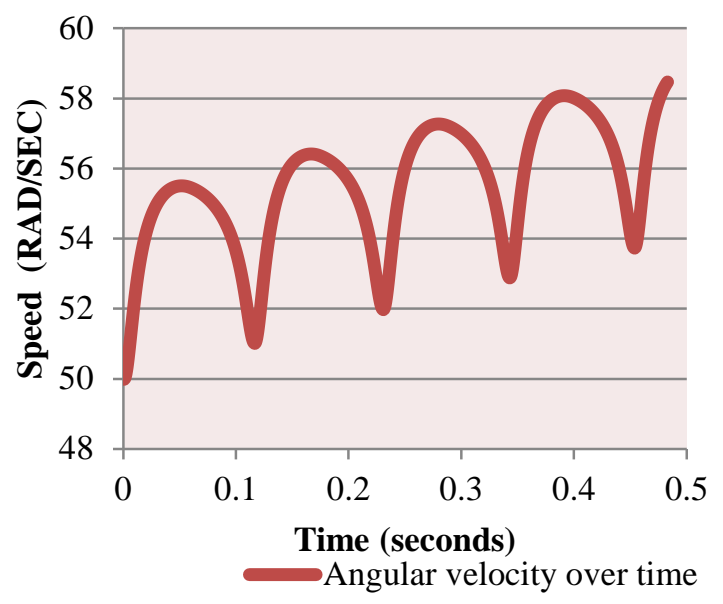

Figure 4: A closer look at Figure 3 shows a sinusoidal wave. 


\section{DISCUSSION}

Applying Euler's modified method for estimating differential equations to the governing equations yields an average angular velocity over time of $72 s^{-1}$ as well as a spot on image of Kranc's graph (Figure 2). The results were replicable, meaning that under the circumstances the information provided is accurate and if the same mathematical methodology is applied someone would reach the same end of a convergence of $72 s^{-1}$ for this theoretical application of an internal combustion engine. Hence, a similar if not equal model of a simplified internal combustion engine was achieved.

\section{CONCLUSION AND RECOMMENDATIONS}

The objective of this project was met by recreating a manageable model of a simplified internal combustion engine to a suitably small approximation using Euler's method for ordinary differential equations. The graph in Figure 3 was able to emulate a close match to the reputable source of data by using sufficiently small intervals. For future reference, this project could be expanded by modifying the system to recreate a four-stroke engine or reciprocating compressor. The efficiency of the engine could also be measured to test what kind of output a simplified model internal combustion engine has under these specific conditions, constants, and variables. Parameters of the project could be changed to show what kind of effect each has on the graph of angular velocity over time, taking note of which variables can vary without affecting the balance of the engine greatly. In a usual case, the steady state equation for a fluid that has a constant flow could have been applied in this project as well. In the end, the machine, the engine in this case, proved to be reliable under some set of conditions which is ideal in our ongoing pursuit of progress. 


\section{NOMENCLATURE}

\begin{tabular}{|c|c|c|}
\hline Symbol & Description & Value/Units \\
\hline$A$ & Piston area & $m^{2}$ \\
\hline C & Load constant & $\mathrm{kgm}^{2}$ \\
\hline$G$ & Constant & - \\
\hline$I$ & Flywheel moment of inertia & $\mathrm{kgm}^{2}$ \\
\hline$K$ & Number of integration steps & $N$ \\
\hline$n$ & Polytropic exponent & Polytropic Index \\
\hline$P$ & Pressure & Pascals or $1 \mathrm{~N} / \mathrm{m}^{2}$ \\
\hline$R$ & Flywheel radius & $m m$ \\
\hline$t$ & Time & seconds \\
\hline$V$ & Volume & $m^{3}$ \\
\hline$T$ & Torque & $(N \cdot m)$ \\
\hline$x_{0}$ & Clearance distance & $\mathrm{mm}$ \\
\hline$\theta$ & Crank angle & radians \\
\hline$\ddot{\theta}$ & Angular acceleration & $(\mathrm{rad} / \mathrm{sec})$ or $\mathrm{s}^{-2}$ \\
\hline$\omega, \omega_{\text {ave }}$ & Angular velocity & $(\mathrm{rad} / \mathrm{sec})$ or $\mathrm{s}^{-1}$ \\
\hline
\end{tabular}




\section{REFERENCES}

Serway, Raymond A., and John W. Jewett. Physics for Scientists and Engineers. 8th ed. Vol. 1. Belmont, CA: Brooks/Cole CENGAGE Learning, 2010.

Stewart, James. Essential Calculus: Early Transcendentals. Belmont, CA: Brooks/Cole, 2011.

Kranc, SC. "A Simplified Model of the Internal Combustion Engine." International Journal of Mechanical Engineering Education (IJMEE) - IMechE \& UMIST 5.4 (1977): 343-46.

Campbell, Scott W. "Project Development." Personal interview. 20 Feb. 2012. 3 May 2012.

Campbell, Scott. Euler's Method. Tampa: University of South Florida, 2012. PDF.

Siyambalapitiya, Chamila. "Project Development." Personal interview. 27 Apr. 2012.

Adkins, William A., and Mark G. Davidson. Ordinary Differential Equations. Berlin: Springer, 2009. www.math.lsu.edu. LSU, 16 Aug. 2009, last access 4 May 2012.

Mattuck, Arthur. "MIT OPENCOURSEWARE." Lecture. Video Lectures. MIT, Massachusetts. 1 May 2012. MIT OPENCOURSEWARE., $<$ http://ocw.mit.edu/courses/mathematics/18-03-differential-equations-spring2010/video-lectures $>$.

Brain, Marshall. "How Two Stroke Engines Work." HowStuffWorks. HowStuffWorks, 07 May 2012.

"Compression and Expansion of Gases." Compression and Expansion of Gases.

Engineeringtoolbox.com, n.d. Web. 01 Apr. 2013. <http://www.engineeringtoolbox.com/> 


\section{APPENDIX}

\begin{tabular}{|ccc|}
\hline Symbol & Value/Unit & Conversion \\
\hline$P_{1}$ & $0.1 \mathrm{MPa}$ & $100,000 \mathrm{~Pa}$ \\
\hline$P_{3}$ & $10.3 \mathrm{MPa}$ & $10,300,000 \mathrm{~Pa}$ \\
\hline$n$ & 1.3 & 1.3 \\
\hline$C$ & $0.0113 \mathrm{kgm}^{2}$ & $0.0113 \mathrm{~kg} \mathrm{~m}^{2}$ \\
\hline$R$ & $305 \mathrm{~mm}$ & $0.305 \mathrm{~m}$ \\
\hline$x_{0}$ & $25.4 \mathrm{~mm}$ & $0.0254 \mathrm{~m}$ \\
\hline$I$ & $3.1710 \mathrm{kgm}^{2}$ & $3.171 \mathrm{~kg} \mathrm{~m}^{2}$ \\
\hline$A$ & $0.00188 \mathrm{~m}^{2}$ & $0.00188 \mathrm{~m}^{2}$ \\
\hline$\theta_{1}$ & $\pi$ & $3.1416 \mathrm{rads}$ \\
\hline$\theta_{2}$ & $2 \pi$ & $6.2832 \mathrm{rads}$ \\
\hline$\theta_{3}$ & 0 & $0.0000 \mathrm{rads}$ \\
\hline$\theta_{4}$ & $\pi$ & $3.1416 \mathrm{rads}$ \\
\hline
\end{tabular}

Table 2: Values of the constants and variables used in the project.

\begin{tabular}{|rcccccccccc|}
\hline Trial & $\boldsymbol{t}$ & $\boldsymbol{\theta}$ & $\boldsymbol{\omega}$ & $\boldsymbol{\theta}^{\prime}$ & $\boldsymbol{\omega}^{\prime} \boldsymbol{a}$ & $\boldsymbol{\omega}^{\prime} \boldsymbol{b}$ & cycle & $\boldsymbol{\theta}$ ref. & $\boldsymbol{\omega}^{\prime}$ & $\begin{array}{c}\boldsymbol{\theta} \\
\text { (Rad) }\end{array}$ \\
\hline $\mathbf{1}$ & 0.000 & 0.0000 & 50.0000 & 50.0000 & -8.9089 & -8.9089 & 0 & 0.0000 & -8.90886 & 0 \\
\hline $\mathbf{2}$ & 0.001 & 0.0500 & 49.9911 & 49.9911 & 49.4403 & 82.54155 & 0 & 0.0500 & 82.54155 & 1 \\
\hline $\mathbf{3}$ & 0.002 & 0.1000 & 50.0736 & 50.0736 & 101.2144 & 163.7052 & 0 & 0.1000 & 163.7052 & 2 \\
\hline $\mathbf{4}$ & 0.003 & 0.1501 & 50.2373 & 50.2373 & 141.9477 & 227.5809 & 0 & 0.1501 & 227.5809 & 3 \\
\hline $\mathbf{5}$ & 0.004 & 0.2003 & 50.4649 & 50.4649 & 169.9542 & 271.5226 & 0 & 0.2003 & 271.5226 & 4 \\
\hline $\mathbf{6}$ & 0.005 & 0.2508 & 50.7364 & 50.7364 & 185.9504 & 296.6494 & 0 & 0.2508 & 296.6494 & 5 \\
\hline $\mathbf{7}$ & 0.006 & 0.3015 & 51.0331 & 51.0331 & 192.0628 & 306.2906 & 0 & 0.3015 & 306.2906 & 6 \\
\hline $\mathbf{8}$ & 0.007 & 0.3525 & 51.3394 & 51.3394 & 190.8609 & 304.4701 & 0 & 0.3525 & 304.4701 & 7 \\
\hline $\mathbf{9}$ & 0.008 & 0.4039 & 51.6439 & 51.6439 & 184.7280 & 294.9213 & 0 & 0.4039 & 294.9213 & 8 \\
\hline $\mathbf{1 0}$ & 0.009 & 0.4556 & 51.9388 & 51.9388 & 175.5839 & 280.6513 & 0 & 0.4555 & 280.6513 & 9 \\
\hline $\mathbf{1 1}$ & 0.01 & 0.5075 & 52.2194 & 52.2194 & 164.8383 & 263.8685 & 0 & 0.5075 & 263.8685 & 10 \\
\hline $\mathbf{1 2}$ & 0.011 & 0.5597 & 52.4833 & 52.4833 & 153.4580 & 246.0876 & 0 & 0.5597 & 246.0876 & 11 \\
\hline $\mathbf{1 3}$ & 0.012 & 0.6121 & 52.7294 & 52.7294 & 142.0681 & 228.2883 & 0 & 0.6122 & 228.2883 & 12 \\
\hline
\end{tabular}

Table 3: Table presents all the relevant data and information used to obtain a replicable graph and approximate angular velocity. 